\title{
Relationship between Disruptions of Carbon's Cyclic Set Natural Transfers and the Upsurge of Weather Conditions with Strong Winds and Heavy Rains
}

\author{
César Mbane Biouele \\ Laboratory of Earth's Atmosphere Physics, Department of Physics, University of Yaoundé I, Yaoundé, \\ Cameroun \\ Email: cesar.mbane@yahoo.fr
}

Received 12 August 2015; accepted 6 October 2015; published 9 October 2015

Copyright (C) 2015 by author and Scientific Research Publishing Inc.

This work is licensed under the Creative Commons Attribution International License (CC BY). http://creativecommons.org/licenses/by/4.0/

(c) (i) Open Access

\section{Abstract}

The cyclic set of natural transfers of carbon (also called: Global Carbon Cycle) is built with the following physicochemical and bacteriological processes: 1) atmosphere to green plants or soil transfers; 2) animals or plants to atmosphere transfers; 3) burning fossil fuels to atmosphere transfers; 4) animals (including humans) to soil transfers; 5) atmosphere to oceans transfers, etc. This whole cycle (despite its obvious complexity), is a formal proof that disturbances recorded by carbon cycle described above, inevitably alter the chemical composition of the atmosphere and, therefore, lead to climate change whose magnitude depends on the sensitivity of the atmosphere relative to the changed settings. The weather models used to assess climate change caused by CFCs, have the annoying tendency to neglect the contribution of clouds to the global carbon balance. Yet it has been proven by $C$. Mbane in 2015 that clouds are composed of only two elements (dry air and saturated water vapor); and that clouds' saturated water vapor may form precipitation (solid or liquid) if and only if they met and dissolve condensation nuclei (mainly the carbon dioxide $\left(\mathrm{CO}_{2}\right)$ in the case of Troposphere). In other words, atmosphere absorbs a significant amount of $\mathrm{CO}_{2}$ in the formation of rains. The purpose of our work is to make it clear to the international opinion that cloud's saturated water vapor is the true lung of our planet, far ahead of the green plants respiration or photosynthesis those take place only under the influence of sunlight and in the very lower layers of the troposphere (e.g., well below 25 meters from the surface of the earth). Each of us could then easily understand the link between GCCD (Global Carbon Cycle Disruptions) and the upsurge of WCWSWHR (weather conditions with strong winds and heavy rains). 


\section{Keywords}

\section{Global Carbon Cycle Disruptions (GCCD), Upsurge of WCWSWHR, Link between GCCD and WCWSWHR}

\section{Introduction}

Carbon is a simple chemical substance (of: 12.01 atomic mass) which exists in its pure form as DIAMOND or GRAPHITE, and is an important part of other substances such as coal and oil, as well as being contained in all plants and animals. Carbon compounds are the basis of all living matter. Carbon dioxide $\left(\mathrm{CO}_{2}\right)$ is the gas formed when carbon is burned, or when animals breathe out: carbon dioxide emissions. Carbon monoxide (CO) is a deadly, colorless, odorless, poisonous gas. It is called the "Invisible Killer" and is produced by the incomplete burning of various fuels, including coal, wood, charcoal, oil, kerosene, propane, and natural gas. Products and equipment powered by internal combustion engines such as portable generators, cars, lawn mowers, and power washers also produce CO: levels of carbon monoxide in the air are worryingly high. Carbon dating is a method of calculating the age of extremely old objects by measuring the amount of Carbon-14 in them. Carbon-14 is a radioactive isotope of carbon mainly produced in the atmosphere. To summarize, the cyclic set of natural transfers of carbon (also called: Global Carbon Cycle) is built with the following physicochemical and bacteriological processes: 1) atmosphere to green plants or soil transfers; 2) animals or plants to atmosphere transfers; 3) burning fossil fuels to atmosphere transfers; 4) animals (including humans) to soil transfers, atmosphere to oceans transfers, etc. This whole cycle (despite its obvious complexity), is a formal proof that disturbances recorded by carbon cycle described above, inevitably alter the chemical composition of the atmosphere and, therefore, lead to climate change whose magnitude depends on the sensitivity of the atmosphere relative to the changed settings. The weather models used to assess climate change caused by CFCs, have the annoying tendency to neglect the contribution of clouds to the global carbon balance. Yet it has been proven by C. Mbane [1] that clouds are composed of only two elements (dry air and saturated water vapor); and that clouds' saturated water vapor may form precipitation (solid or liquid) if and only if they met and dissolve condensation nuclei (mainly the carbon dioxide $\left(\mathrm{CO}_{2}\right)$ in the case of Troposphere). In other words, Atmosphere absorbs a significant amount of $\mathrm{CO}_{2}$ in the formation of rains. The purpose of our work is to make it clear to the international opinion that cloud's saturated water vapor is the true lung of our planet, far ahead of the green plants respiration or photosynthesis those take place only under the influence of sunlight and in the very lower layers of the troposphere (e.g., well below 25 meters from the surface of the earth). Each of us could then easily understand the link between GCCD (Global Carbon Cycle Disruptions) and the upsurge of WCWSWHR (weather conditions with strong winds and heavy rains). Given the fact that planetary production of saturated water vapor is not an experience of the reach of humans' laboratories, the reduction of CFCs emissions and the forestation of ground's surface (or increasing the green canopy) remains the only solution to save the planet from global warming and the upsurge of large atmospheric disturbances.

\section{Urgent Need of Faithful Representations of Cyclic Set of Natural Transfers of Carbon}

Many representations of the global carbon cycle have been proposed (Figure 1 and Figure 2). However, the information they provide remains incomplete because nowhere in these illustrations, the real contribution of clouds in destroying atmosphere's CFCs is objectively estimated in a clear and precise manner. Ignorance of physical properties of the cloud is largely responsible for this neglect that, historically, led to the production of totally ineffective climate models. Introduction of arrows showing $\mathrm{CO}_{2}$ absorption by clouds (necessary processes leading to rains), into Figure 1 and Figure 2, would greatly contribute to their improvement. It is very important to know that plants' canopy photosynthesis and respiration are diurnal processes, while clouds destruction of CFCs occurs both by day or night. Therefore, cloud's saturated water vapor is the biggest lung of our planet; far ahead of the smaller green-lung related to plants respiration or photosynthesis those take place only under the influence of sunlight and in the very lower layers of the troposphere (e.g., plants' canopy altitudes). 


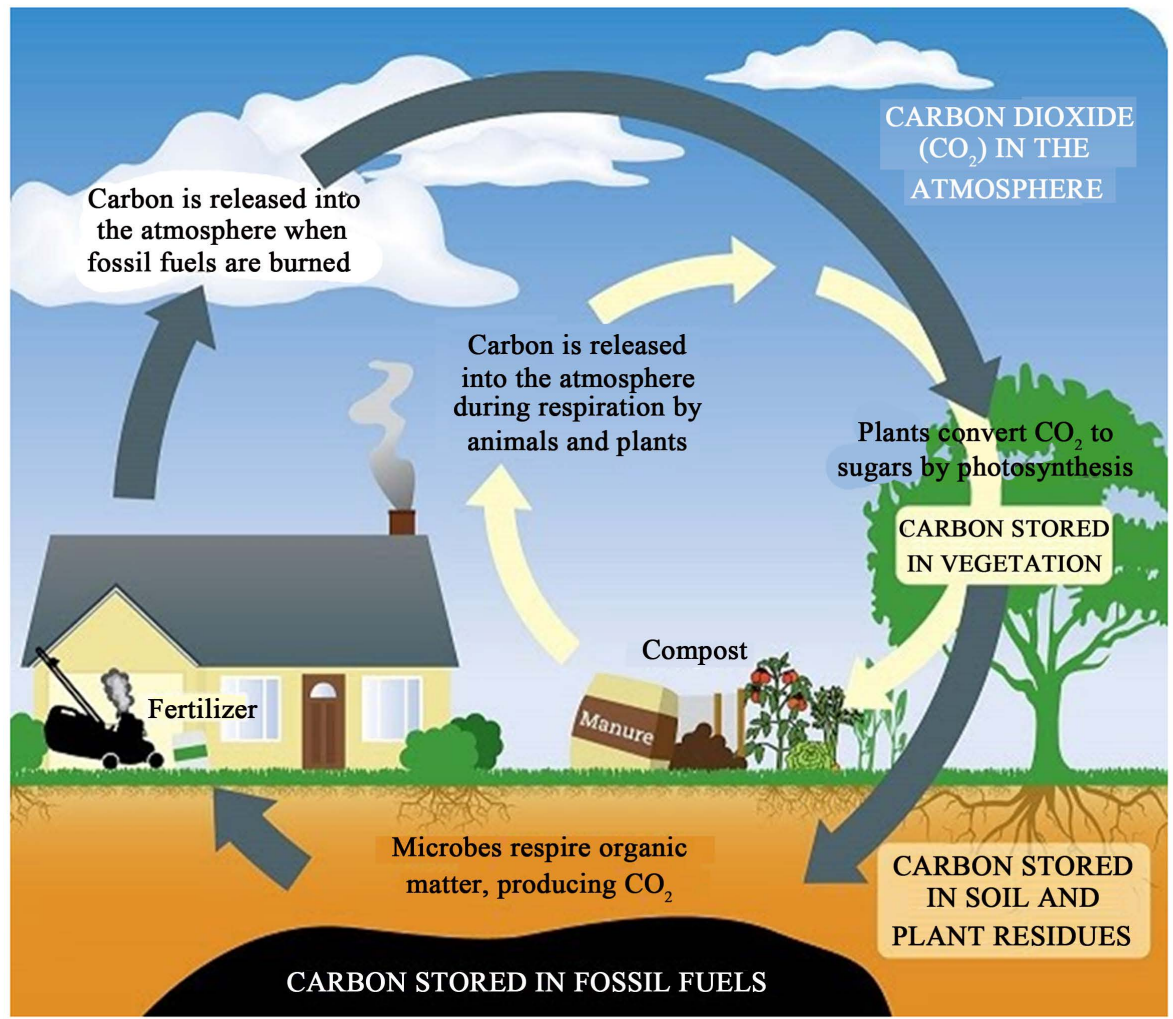

Figure 1. Global carbon cycle: rains bring carbonates to soil.

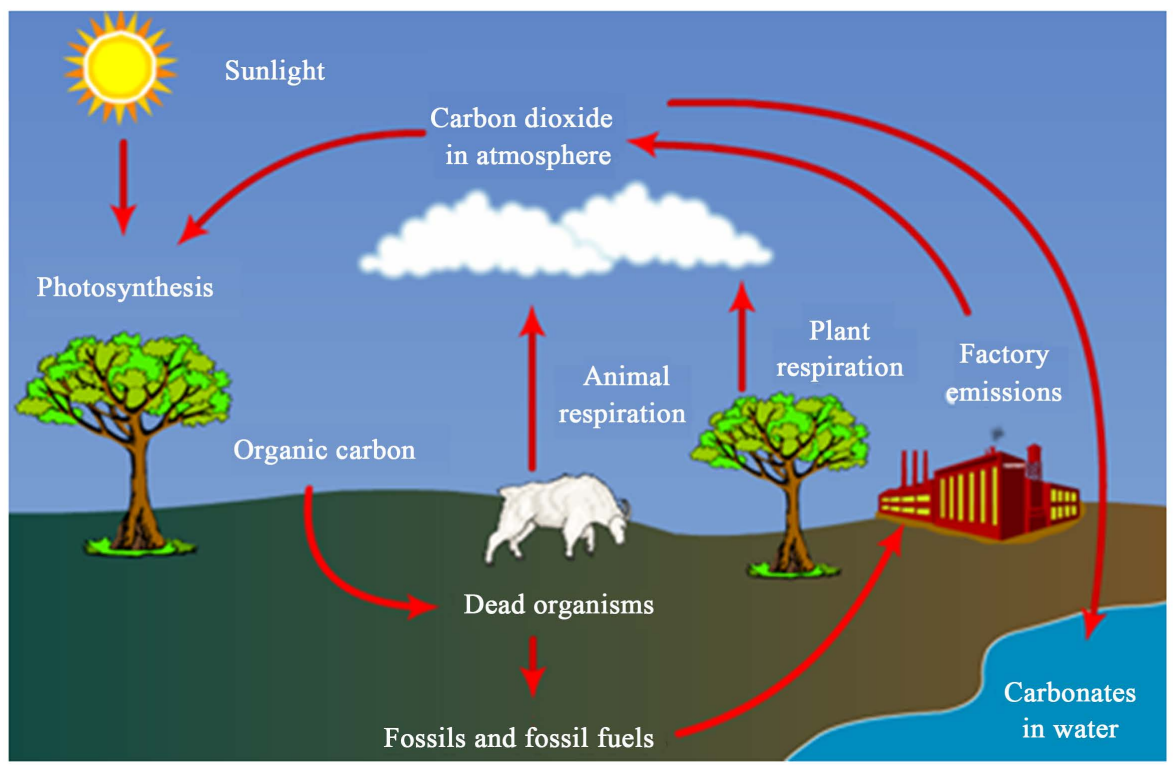

Figure 2. Global carbon cycle: rains bring carbonates to oceans.

\section{Cloud's Saturated Water Contribution to Global Carbon Cycle}

As demonstrated by C. Mbane Biouele, precipitation formation occurs under specific thermodynamic conditions. Cloud's saturated water vapor, reacts chemically with condensation nuclei to form droplets (solid or liquid) which (fatally) fall under the gravity effects. Clouds are consequently a region of very short transit for the water droplets. In Figure 3, it is clear that the clouds occupy three floors of the Troposphere. This makes it possible to 


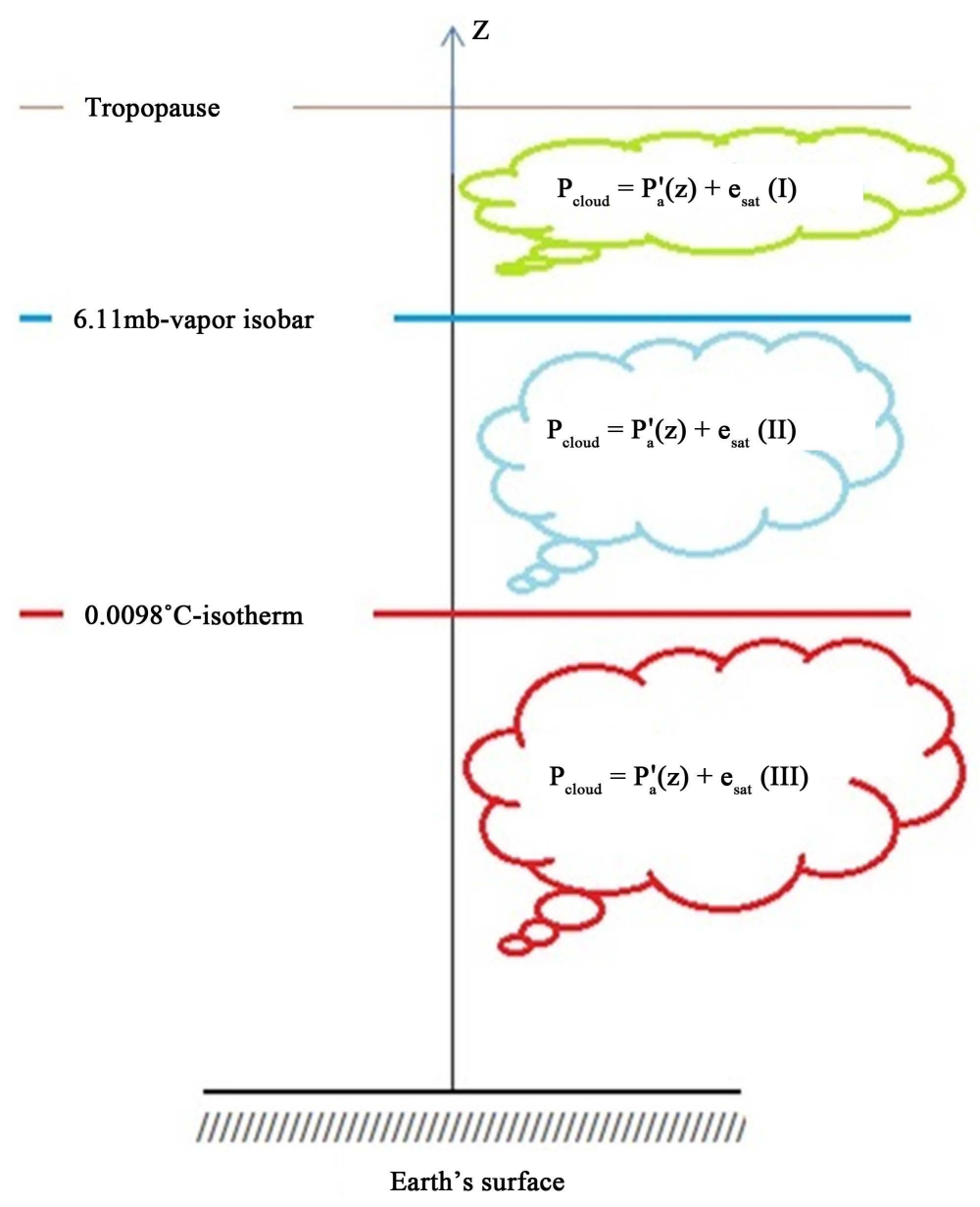

Figure 3. Cloud's saturated water vapors destroy $\mathrm{CO}_{2}$ at all troposphere's levels.

effectively destroy the CFCs in these three different floors. Hence the effectiveness of their destroying work compared to work done by photosynthesis and plant respiration processes. A perfect knowledge [2] of the earth's atmosphere general circulation (Figure 4) allows us to understand that, as occurs in the human body, the streamlines of this atmospheric circulation are the arteries that connect the lungs (green lung with respect to plants, and grey lung with respect to clouds) to the rest of the body (the whole atmosphere in this case). Given the high evaporation triggered by Global Warming, precipitation intensity increases logically with atmospheric pollution levels. This correlation is now clearly established and should encourage Authorities of this world to avoid the upsurge of rainy storms whose harmful effects are irreversible as what is happening these recent days in India. Reducing emissions of CFCs is a mandatory way to save our planet.

\section{What Else Must We Learn on the Usefulness and Fragility of Earth's Atmosphere?}

Nature has generously offered to our planet, two huge “nuclear barriers” that protect the Earth (one of the sun's satellites) against the perverse effects produced by solar wind (Figure 5) and UV (or IR) radiation (Figure 6). These huge barriers are the magnetosphere, which deflects the solar wind from its original path oriented towards the Earth and the atmosphere that filters the UV and IR rays (Figure 6). Given what has just been said clearly, changing the chemical composition of the atmosphere by providing carelessly, many pollutants in it, lead fatally to a change of its selective properties (e.g., the crossing of the ozone layer by UV rays, through the holes inappropriately opened in this savior layer). Sufficiently efficient studies should be conducted to serve as support tools for the decision to the authorities of this world. Because most of them find it very difficult to understand: earth's atmosphere usefulness and fragility. It is therefore necessary to convince all people across the planet 


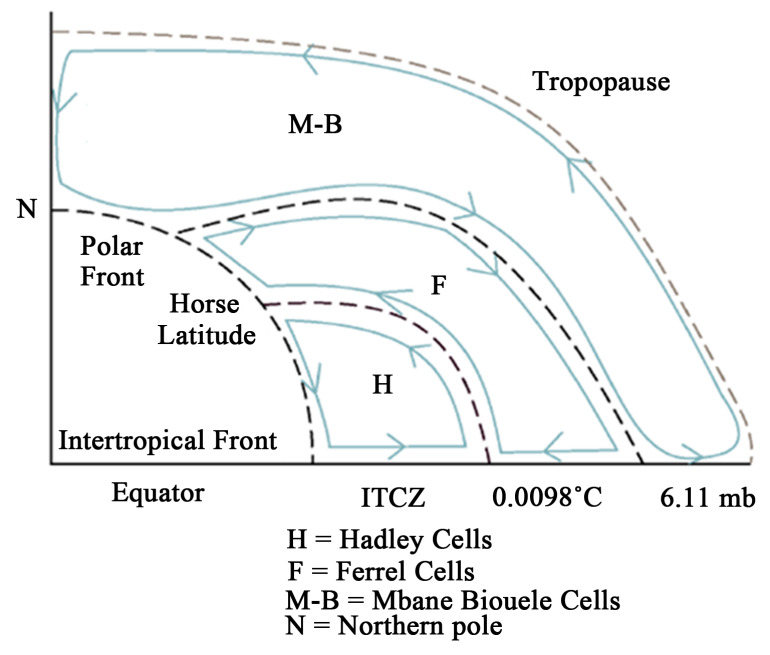

Figure 4. Unique and faithful representation of Earth's atmosphere general circulation [2].

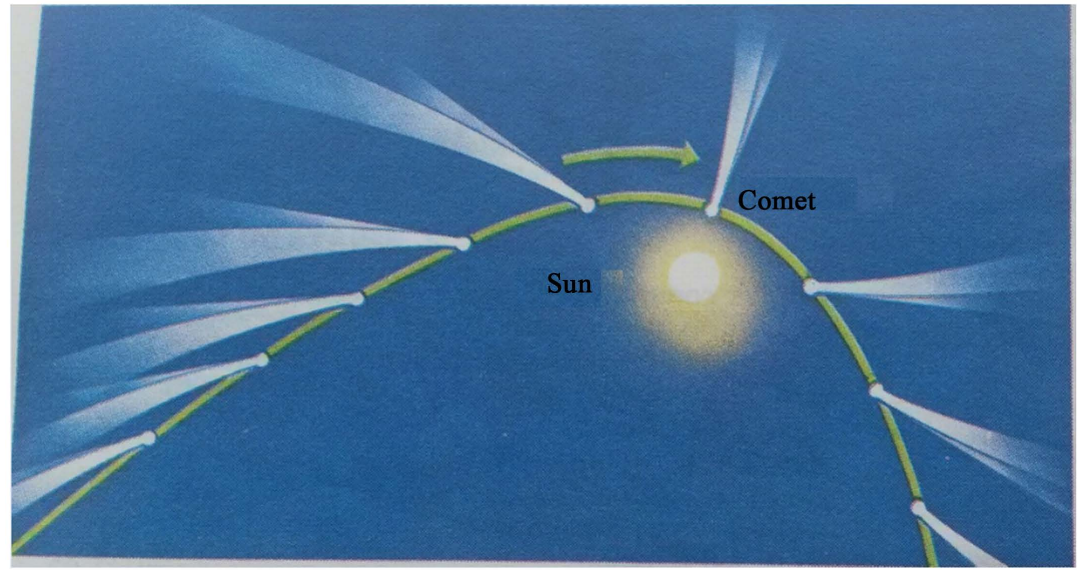

Figure 5. Impacts of Solar wind on Comet. Elementary particles $(\alpha, \beta, \gamma$, etc.) carried by the violent solar wind collide with the comet and ionize (or burn); then they form what is considered wrongly as a drag (or tail) of the comet.

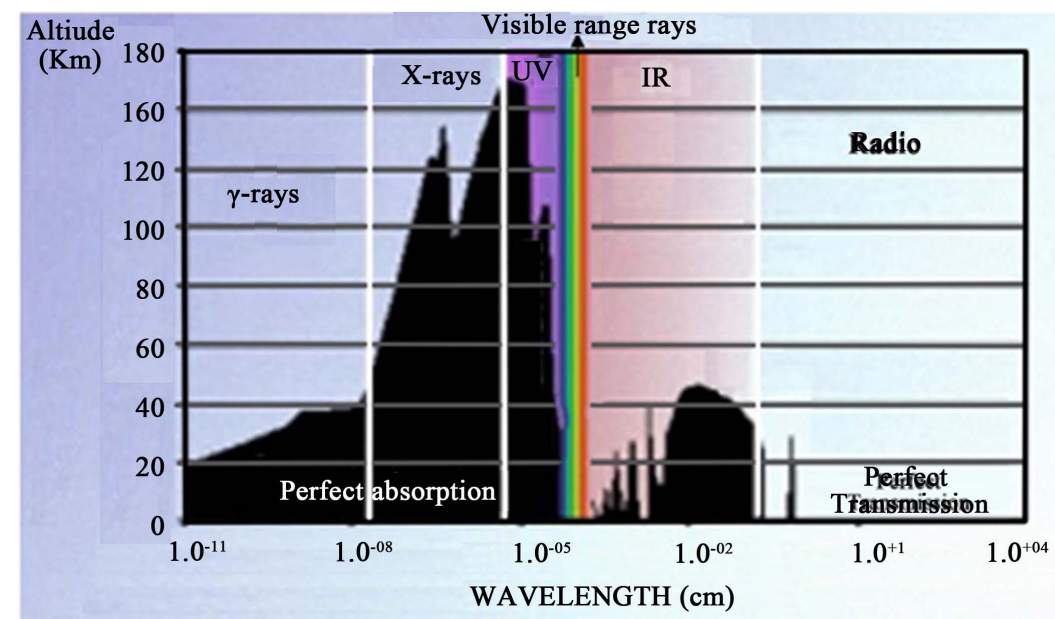

Figure 6. Selective transparency properties of earth's atmosphere. 
that earth's atmosphere (dwelling place of clouds) is both a huge lung (e.g., it removes $\mathrm{CO}_{2}$ ) and filter (e.g., it stops the IR and UV rays).

\section{Conclusion}

The purpose of our work was to make it clear to the international opinion that cloud's saturated water vapor is the true lung of our planet, far ahead of the green plants respiration or photosynthesis those take place only under the influence of sunlight and in the very lower layers of the troposphere (e.g., well below 25 meters from the surface of the earth). Each of us may now easily understand the link between GCCD (Global Carbon Cycle Disruptions) and the upsurge of WCWSWHR (weather conditions with strong winds and heavy rains). Given the fact that planetary production of saturated water vapor is not an experience of the reach of humans' laboratories, the reduction of CFCs emissions and the forestation of ground's surface (or increasing the green canopy) are mandatory ways to save our planet.

\section{References}

[1] Mbane Biouele, C. (2015) Fundamentals on Thermodynamic Processes behind Cloud's and Rainfall's Formation. Atmospheric and Climate Sciences, 5, 257-265. http://dx.doi.org/10.4236/acs.2015.53019

[2] Mbane Biouele, C. (2015) Earth’s Atmosphere Dynamic Balance Meteorology. Scientific Research Publishing Inc., $110 \mathrm{p}$. 